\title{
MONOMIAL EQUIMULTIPLE CURVES IN POSITIVE CHARACTERISTIC
}

\author{
R. NARASIMHAN
}

\begin{abstract}
It is known that the local equimultiple locus of a hypersurface in characteristic zero is contained in a regular hypersurface. Here we give an example of a monomial curve on a threefold in positive characteristic $p>2$ which is equimultiple but not hyperplanar. As a corollary we have that any monomial curve which lies on a certain type of hypersurface (whose local equation is of a special form in its natural $p$-basis expression) is automatically equimultiple for the hypersurface.
\end{abstract}

Introduction. Consider the hypersurface $F \in k\left[\left[X_{1}, \ldots, X_{n}, Z\right]\right]$ whose local equation is

$$
F=Z^{d}+a_{1} Z^{d-1}+\cdots+a_{d}
$$

such that $a_{i} \in k\left[\left[X_{1}, \ldots, X_{n}\right]\right]$ and ord $F=d$. Let $P$ be a prime ideal in $k\left[\left[X_{1}, \ldots, X_{n}, Z\right]\right]$ such that $F \in P^{(d)}$ (the $d$ th symbolic power of $P$ ). Then since

$$
D F \in P^{(d-1)} \text { for any derivation } D \text { on } k\left[\left[X_{1}, \ldots, X_{n}, Z\right]\right] \text {, }
$$

taking $(d-1)$ partial derivatives with respect to $Z$ we get

$$
D_{Z}^{(d-1)}(F)=d ! Z+a_{1}(d-1) ! \in P .
$$

If char $k=0$, this may be interpreted as saying that the equimultiple curve $P$ is contained in the hyperplane (i.e. regular hypersurface) $Z+a_{1} / d=0$. (See Abhyankar's paper [A] for details.)

In positive characteristic it is known that any equimultiple curve on a surface is hyperplanar. [Some cases of this were done in an earlier paper [N]; a general 'characteristic-free' proof was given recently by S. B. Mulay (to appear).] In [N] we gave an example in characteristic two showing that an equimultiple curve on a variety of dimension bigger than two need not be hyperplanar. In this paper we give an example of a (3-dimensional) variety in characteristic $p<2$ having an equimultiple curve which is not hyperplanar.

We wish to thank Avinash Sathaye and Jean Giraud for their helpful suggestions. We are also extremely grateful to Professor S. S. Abhyankar for his guidance and kind encouragement.

Received by the editors January 12, 1983.

1980 Mathematics Subject Classification. Primary 14B05; Secondary 13H05, $13 \mathrm{H} 15$.

Key words and phrases. Equimultiple curve, ideal-theoretic complete intersection, symmetric semigroup. 
The main aim of this paper is to prove the following

THEOREM. Let $k$ be an algebraically closed field of characteristic $p>2$. Let $A=k[[X, Y, Z, W]]$, and let $P=\operatorname{ker} \phi$ where $\phi$ is given by

$$
\begin{array}{lrl}
A \stackrel{\phi}{\rightarrow} k[[t]] & (t \text { an indeterminate }), \\
X \mapsto t^{a_{1}} & \text { with } a_{1}=(2 p-2)(2 p+1), \\
Y \mapsto t^{a_{2}} & a_{2}=p(2 p+1), \\
Z \mapsto t^{a_{3}} & a_{3}=p(2 p-1), \\
W \mapsto t^{a_{4}} & a_{4}=c-1,
\end{array}
$$

where $c$ is the conductor of the semigroup $\left\langle a_{1}, a_{2}, a_{3}\right\rangle$. Then there exists a hypersurface defined by

$$
F=W^{p}+\sum_{\substack{0 \leqslant i, j \leqslant p-1 \\(i, j) \neq(0,0)}}\left(f_{i j}(X, Y, Z)\right)^{p} Y^{i} Z^{j}
$$

such that

(i) $P$ is an equimultiple curve on $F$, i.e., $\operatorname{ord}_{A} F=\operatorname{ord}_{A_{P}} F A_{P}=p$;

(ii) $P$ does not contain a regular parameter of $A$.

The proof will follow from the following lemmas.

LEMma 1. With the same notation as above, there exist $f_{i j}$ for all $i, j$ with $0 \leqslant i, j \leqslant p-1,(i, j) \neq(0,0)$ satisfying the following conditions:

(a) ord ${ }_{t}\left(\phi\left(f_{i j}\right)\right) \in\left\langle a_{1}, a_{2}, a_{3}\right\rangle$,

(b) $f_{i j} \neq 0$,

(c) $\phi(F)$ is homogeneous (in $k[t])$.

(REMARK. (a) implies the $f_{i j}$ 's can, in fact, be chosen to be monomials in $X, Y, Z$.)

Proof. Let $Q=P \cap k[[X, Y, Z]]$. Let $x, y, z$ denote the residues of $X, Y, Z$ modulo $P$. We note that the numbers $a_{1}, a_{2}, a_{3}$ satisfy the following conditions:

(i) $\left(a_{1}, a_{2}, a_{3}\right)=1$. (In fact, since $a_{1}=2 a_{3}-2$ and $a_{3}$ is odd, $\left(a_{1}, a_{3}\right)=1$.)

(ii) $\left[\left(a_{1}, \ldots, a_{i}\right), a_{i+1}\right] \in\left\langle a_{1}, \ldots, a_{i}\right\rangle$ for $i=1,2$. (As usual, $\left(a_{1}, \ldots, a_{n}\right)$ denotes the g.c.d., and $\left[a_{1}, \ldots, a_{n}\right]$ denotes the l.c.m. of $a_{1}, \ldots, a_{n}$.)

Hence we may apply Proposition 2.1 of $[\mathbf{H}]$ to conclude that:

(i)' $Q$ is an ideal-theoretic complete intersection. (This also follows by observing that $X^{p}-Y^{2 p-2}$ is a minimal relation in $Q$ in the sense of [H].) Hence

(ii)' The semigroup $\left\langle a_{1}, a_{2}, a_{3}\right\rangle$ is symmetric.

(iii)' The conductor $c$ of $S$ is given by

$$
\begin{aligned}
a_{4} & =c-1=\sum_{i=1,2}\left[\left(a_{1}, \ldots, a_{i}\right), a_{i+1}\right]-\sum_{i=1}^{3} a_{i} \\
& =8 p^{3}-10 p^{2}-p+2 .
\end{aligned}
$$

Now the condition for $F$ to be weighted homogeneous is

$$
p a_{4}=p\left[\operatorname{ord}_{t} \phi\left(f_{i j}\right)\right]+i a_{2}+j a_{3},
$$


i.e.

$$
a_{4}=\operatorname{ord}_{t} \phi\left(f_{i j}\right)+i(2 p+1)+j(2 p-1) .
$$

Hence to prove the existence of the $f_{i j}$ 's, using (ii)' and (iii)' above, we need only prove the following.

Claim. $i(2 p+1)+j(2 p-1) \notin S$ for $0 \leqslant i, j \leqslant p-1,(i, j) \neq(0,0)$.

Proof of Claim. Suppose, if possible,

$$
i(2 p+1)+j(2 p-1)=\lambda a_{1}+\mu a_{2}+\nu a_{3}, \quad \lambda, \mu, \nu \geqslant 0 .
$$

Then

$$
\max _{0 \leqslant i, j \leqslant p-1} i(2 p+1)+j(2 p-1)=(p-1)(4 p)<4 p^{2}-2 p-2=a_{1}
$$

gives $\lambda=0$. Thus

$$
i(2 p+1) j(2 p-1)=\mu p(2 p+1)+\nu p(2 p-1) \Rightarrow p \mid(i-j) .
$$

But $0 \leqslant i, j \leqslant p-1 \Rightarrow|i-j| \leqslant p-1$. Thus $i=j \neq 0$ and $2 i+2 j=\mu(2 p+1)+\nu(2 p-1) \Rightarrow 4 i=\mu(2 p+1)+\nu(2 p-1), \quad i \leqslant p-1$. Clearly $(\mu, \nu)=(1,0)$ or $(0,1)$ is impossible and $\mu \geqslant 1$ or $\nu \geqslant 1 \Rightarrow \mu(2 p+1)+$ $\nu(2 p-1) \geqslant 4 p-2$, contradiction. This proves the Claim, and since, in (I),

$$
\begin{aligned}
a_{4}-\left[\max _{0 \leqslant i, j \leqslant p-1} i(2 p+1)+j(2 p-1)\right] & \geqslant a_{4}=p(4 p), \\
& >0 \text { when } p \geqslant 2 \text { by (iii) }{ }^{\prime},
\end{aligned}
$$

we get $f_{i j} \neq 0$ for all the relevant $i$ and $j$. This completes the proof of Lemma 1 .

Lemma 2. Let $A=k[[X, Y, Z, W]]$. Let $P \in \operatorname{Spec} A$ be such that $\operatorname{dim} A / P=1$; let $k[[t]]$ denote the integral closure of $A / P$ in its quotient field. Let $x, y, z$ and $w$ denote the residues of $X, Y, Z$ and $W$ modulo $P$. Assume $x=t^{a_{1}}, y=t^{a_{2}}, z=t^{a_{3}}, w=t^{a_{4}}$ for some positive integers $a_{1}, a_{2}, a_{3}, a_{4}$. Let $F=W^{p}+f(X, Y, Z)$, where

$$
f(X, Y, Z)=\sum_{\substack{0 \leqslant i, j \leqslant p-1 \\(i, j) \neq(0,0)}} f_{i j}^{p} Y^{i} Z^{j}
$$

such that

(a) the $f_{i j}$ are nonzero monomials in $X, Y, Z$ and $f_{i j}(1,1,1)=1$,

(b) $F$ is weighted homogeneous (with weights $a_{1}, a_{2}, a_{3}, a_{4}$ for $X, Y, Z$ and $W$ ).

Then $F \in P^{(p)}$.

Proof. Let $D_{i j}$ denote the (usual) partial derivatives with respect to $Y$ and $Z$. Then $F \in P^{(p)} \Leftrightarrow D_{i j}(F) \in P$ for $0 \leqslant i+j \leqslant p-1$. (For a proof of this, see Lemma 4.3.3(i) of $[\mathbf{G}]$.) By hypothesis (conditions (a) and (b)) $P$ lies on $F$, i.e. $D_{00}(F) \in P$.

Now $F(X, Y, Z, W)$ is weighted homogeneous. Hence all derivatives $D_{i j}(F)$ are again weighted homogeneous. (This observation is independent of the special forms of $F$ and $f_{i j}$ 's.) Hence

$$
D_{i j}(F) \in P \text { for } 0<i+j \leqslant p-1 \Leftrightarrow \text { the sum of the coefficients of } D_{i j}(f)
$$


(in the given form, i.e., in the expansion of $D_{i j}(f)$ with respect to the usual $p$-basis $\left.\left\{Y^{i} Z^{j}\right\}_{0 \leqslant i, j \leqslant p-1}\right)$ equals zero $(\bmod p)$.

But to prove this statement we may further assume $f_{i j}=1$ for $0 \leqslant i, j \leqslant p-1$, $(i, j) \neq(0,0)$, i.e.

$$
\begin{aligned}
f & =\sum_{\substack{0 \leqslant i, j \leqslant p-1 \\
(i, j) \neq(0,0)}} Y^{i} Z^{j}=\left(1+Y+\cdots+Y^{p-1}\right)\left(1+Z+\cdots+Z^{p-1}\right)-1 \\
& =(1-Y)^{p}(1-Z)^{p}(1-Y)^{-1}(1-Z)^{-1}-1 .
\end{aligned}
$$

Now, the sum of the coefficients of $D_{i j}(f)$

$$
=\left.D_{i j}(f)\right|_{\text {evaluated at }(Y, Z)=(1,1)}
$$

and this is obviously zero for

$$
0<i+j<2 p-2
$$

This proves Lemma 2.

Proof of the Theorem. Using Lemma 1 we choose $f_{i j}(X, Y, Z)$ to be monomials in $X, Y, Z$ satisfying the condition $f_{i j}(1,1,1)=1$ (besides the conditions of the lemma). Then Lemma 2 shows that $P$ is an equimultiple curve on $F$. To see that $P$ is not contained in a regular hypersurface we only have to observe that, as in [N], among the four integers $a_{1}, a_{2}, a_{3}, a_{4}$, none is contained in the semigroup generated by the other three. Q.E.D.

ReMARKS. Lemma 2 is easily seen to be equivalent to a 'binomial identity' in characteristic $p>0$. The advantage of our formulation and method of proof is that it extends to several variables. Thus we have the following general property of monomial equimultiple curves on hypersurfaces in higher dimensions:

Proposition. Let $A=k\left[\left[X_{1}, \ldots, X_{n}, W\right]\right]$ with $n \geqslant 2$. Let $P \in \operatorname{Spec} A$ be such that $\operatorname{dim} A / P=1$; let $k[[t]]$ denote the integral closure of $A / P$ in its quotient field. Let $x_{i}$ and $w$ denote the residues of $X_{i}$ and $W$ modulo $P$, for $i=1,2, \ldots, n$.

Let $F=W^{\prime \prime}+f\left(X_{1} \ldots, X_{n}\right)$ where

$$
f\left(x_{1} \ldots x_{n}\right)=\sum_{0 .<r_{1}} f_{1}^{\prime \prime} x^{\prime}
$$

with $X \subseteq\left(X_{1}, \ldots, X_{n}\right)$, card $\left.X\right) \geqslant 2$ and $0<i \ll p$ denotes, as usual, a restricted vector $\left(i_{1}, \ldots, i_{m}\right)$,

$$
0 \leqslant i_{j} \leqslant p-1 \quad \text { for } j=1,2, \ldots, m, \quad\left(i_{1}, \ldots, i_{m}\right) \neq(0, \ldots, 0) .
$$

Assume:

(a) $x_{i}=t^{a_{i}}, w=t^{\omega}$ for some positive integers $a_{i}, \omega, i=1,2, \ldots, n$.

(b) $f_{i}$ 's are nonzero monomials in $X_{1}, \ldots, X_{n}$ with coefficient 1 .

(c) $P$ lies on $F$.

Then $P$ is equimultiple for $F$.

Proof. The proof of Lemma 2 obviously generalises to this situation; observe that the restriction $\operatorname{card}(X) \geqslant 2$ is essential for the validity of the final step (II) in the proof. 


\section{REFERENCES}

[A] S. Abhyankar, Good points on a hypersurface, Adv. in Math. (to appear).

[G] J. Giraud, Contact maximal en caracteristique positive, Ann. Sci. École Norm. Sup. (4) 8 (1975), 201-234.

[H] J. Herzog, Generators and relations of Abelian semigroups and semigroup rings, Manuscripts Math. 3 (1970), 175-193.

[N] R. Narasimhan, Hyperplanarity of the equimultiple locus, Proc. Amer. Math. Soc. 87 (1983), 403-408.

Department of Mathematics, Poona University, Pune 411007, India 